\title{
The prevalence of PAX2 mutations in patients with isolated colobomas or colobomas associated with urogenital anomalies
}

\author{
H E Cunliffe, L A McNoe, T A Ward, K Devriendt, H G Brunner, M R Eccles
}

\begin{abstract}
The PAX2 gene is mutated in patients with ocular colobomas, vesicoureteral reflux (VUR), and kidney anomalies (renalcoloboma syndrome, OMIM 120330). The three abnormalities which make up this syndrome also occur in isolation, but the causal genes are not known. PAX2 encodes a transcription factor of the paired box class of DNA binding proteins, important for the development of the urogenital tract, optic nerve and adjacent retina, inner ear, and CNS. In this paper we have investigated the prevalence of PAX 2 mutations in patients with ocular colobomas, microphthalmos, or retinal anomalies, either in isolation or with associated urogenital anomalies. Using PCR-SSCP, most or all exons of PAX2 were examined in blood DNA from 99 patients who have either ocular anomalies alone or a combination of ocular and urogenital conditions. PAX2 mutations were not detected in patients with ocular colobomas, either in isolation or with associated abnormalities, except in one patient with typical renal-coloboma syndrome. We conclude that PAX2 mutations are unlikely to be common in patients with ocular colobomas in isolation or in patients with ocular colobomas and associated anomalies, except for patients with typical renalcoloboma syndrome where PAX 2 is known to be the aetiological cause.

( $F$ Med Genet 1998;35:806-812)
\end{abstract}

Keywords: PAX2; renal-coloboma syndrome; coloboma; vesicoureteric reflux

Ocular colobomas (gaps or fissures in the structures of the eye) are a relatively common malformation which can occur either sporadically or as an autosomal dominantly inherited malformation involving the iris, retina, choroid, or optic nerve. ${ }^{1}$ The incidence of coloboma in the population is unknown. Colobomas may occur as isolated malformations or as part of a complex multisystem syndrome. ${ }^{1}$ Typically, colobomas result from failure of fusion of the embryonic fissure of the optic cup anywhere along the line extending from the optic disc to the inferonasal border of the pupillary margin. For the purposes of the present paper we shall define the term coloboma to include lesions affecting the optic nerve (ranging from optic disc dysplasia to optic nerve aplasia), retina, choroid, and iris, but not the eyelids. Orbital cysts and microphthalmia are also considere $\$$ to be part of the spectrum of colobomatou» defects resulting from failure of fusion of the embryonic fissure in the optic cup. Rarely, colobomas are reported in association withu other developmental anomalies. For example renal-coloboma syndrome (MIM 120330) is rare autosomal dominant syndrome involving optic nerve coloboma in association with renat anomalies. ${ }^{2-7}$ Other syndromes that may als $\Theta_{0}$ represent coloboma association syndromes include CHARGE association (coloboma heart defects, choanal atresia, retarded growthor development, genital hypoplasia, and eaf anomalies), ${ }^{8}$ Joubert related syndromes such as Dekaban syndrome (chorioretinal colobom£ with cerebellar vermis aplasia and polycystic kidneys), ${ }^{9} \mathrm{COACH}$ syndrome (cerebellar ver mis hypo/aplasia, oligophrenia, congenitąt ataxia, coloboma, hepatic fibrosis), ${ }^{10}$ acro-renoo ocular syndrome, ${ }^{11}$ Sorsby syndrome, branchio-oculo-facial syndrome, ${ }^{13}$ cat ey@ syndrome, ${ }^{14}$ and $3 \mathrm{C}$ syndrome. ${ }^{15}$

We have previously documented mutation of the PAX2 gene in 11 subjects wit? renal-coloboma syndrome, including a family with autosomal dominant optic nerve coloe boma (malformation of the optic nerve), rena anomalies, and vesicoureteral reflum (VUR). ${ }^{16-19}$ Recently we found that PAX mutations may result in highly variable phenotypes. ${ }^{19}$ Since phenotypic variability is feature of this syndrome we hypothesised that PAX2 mutations may sometimes cause sporadic ocular colobomas in isolation in patient The specific genes involved in causing sporadie or hereditary colobomas are not knowns although hypoplasia or agenesis of the fovea of macula have been reported in some patientt్టు with PAX6 mutations. ${ }^{21}$

PAX2 is a member of a multigene famil\$ containing a paired box domain that was initially identified in Drosophila and subse? quently in vertebrates. ${ }^{22}$ PAX2 expression i critical for development of the urogenital trace CNS, inner ear, and optic nerve, ${ }^{23-25}$ as has been reported in mice with kidney and retina defects $(K r d),{ }^{26} \mathrm{Pax}_{2}{ }^{\mathrm{Neu}}$ mice, ${ }^{27}$ and in mice with a Pax2 gene disruption induced bo homologous recombination. ${ }^{28}{ }^{29}$ To investigat whether PAX2 mutations occur more generalls. in patients with colobomas, microphthalmia, of retinal anomalies, or in patients with coloboma associated syndromes, we have analysed patient DNA by PCR-SSCP for PAX2 mutations in subjects who have either ocular anomalies alone, a recognised syndrome such as Dekaban 
syndrome, or a loosely defined combination of ocular and urogenital conditions. Following analysis of 99 patients in most or all exons of PAX2, we have identified one new patient with a PAX2 mutation, who had typical renalcoloboma syndrome (optic nerve colobomas, renal anomalies, and vesicoureteric reflux). We did not identify PAX2 mutations in patients with ocular colobomas or microphthalmia in isolation or in patients with ocular colobomas and associated anomalies.

\section{Subjects and methods}

CLASSIFICATION OF THE PATIENTS IN THE STUDY The patients analysed in this study were divided into five categories: (A) patients with typical renal-coloboma syndrome (optic nerve colobomas and renal hypoplasia, with or without vesicoureteric reflux), (B) patients with optic nerve or chorioretinal colobomas, combined with other anomalies, some of whom had a known syndrome, (C) patients with eye anomalies (not optic nerve or chorioretinal coloboma) in combination with other anomalies, who did not fit a known syndrome, (D) patients with isolated renal anomalies, and (E) patients with predominantly ocular anomalies in isolation. Patients in categories A-D are listed in table 1. Patients in category $\mathrm{E}$ are listed separately under different headings in table 2. Patients were evaluated by history, physical examination, ophthalmological examination, and renal function tests including routine urine and blood analyses and/or renal ultrasound, intravenous pyelogram, or micturating cystourethrogram

PCR-SSCP AND DETECTION OF PAX2 MUTATIONS Genomic DNA from each subject was extracted from peripheral blood using a DNA extraction kit (Promega). Fragments spanning exons 1-12 and the promoter of PAX2 were amplified from genomic DNA using PCR primers flanking the regions of interest (table 3 ). The primer sequences used for amplifying each exon of PAX2 were based on the genomic sequences flanking each exon. ${ }^{30}$ Amplification conditions are given in table 3 . The PCR products of exons 1-10 and the promoter were labelled by incorporation of ${ }^{32} \mathrm{P}$-dCTP in reactions containing $100 \mathrm{ng}$ DNA, $62.5 \mathrm{mmol} / 1$ dNTPs, $1 \mathrm{mCi} \alpha^{32} \mathrm{P}$-dCTP $(3000 \mathrm{Ci} / \mathrm{mmol})$, $20 \mathrm{pmol}$ of each primer, reaction buffer $(50$ $\mathrm{mmol} / 1 \mathrm{KCl}, 10 \mathrm{mmol} / 1$ Tris, $\mathrm{pH} \mathrm{8.3,1.0-3.0}$ $\mathrm{mmol} / \mathrm{l} \mathrm{MgCl}_{2}$ ), and Hot Tub (Amersham) or Pwo (Boehringer) polymerase. Exons 11 and 12 were labelled by kinasing unlabelled PCR products with polynucleotide kinase (New England Biolabs) and $\gamma^{32}$ P-ATP. The PCR products were electrophoresed on nondenaturing $6 \%$ polyacrylamide gels ${ }^{31}$ with $1 \times$ TBE, $\mathrm{pH} 8.0$, buffer to show single strand conformational (SSCP) variants. ${ }^{32}$ Several conditions of polyacrylamide gel electrophoresis were used, including gels with or without $10 \%$ glycerol, and electrophoresis at $25^{\circ} \mathrm{C}$ or at $4^{\circ} \mathrm{C}$. The PCR product from the promoter region was digested with SmaI restriction enzyme
(Promega) to yield three fragments of 325, 233 , and $232 \mathrm{bp}$, which were resolved on SSCP gels.

SUBCLONING AND DNA SEQUENCING

PCR products were directly sequenced using $\alpha^{32} \mathrm{P}-\mathrm{dCTP}$ and a cycle sequencing kit (GIBCO-BRL). For subcloning of exon 2 PCR products, mutant and normal alleles of PAX2 were amplified using Pwo DNA polymerase (Boehringer Mannheim), and subcloned into EcoRV digested pBluescript II (Stratagene). Positive clones were identified by blue/white selection, and plasmid DNA was isolated using Wizard mini-preps kits (Promega). Sequencing reactions were carried out on plasmid DNA using ${ }^{35} \mathrm{~S}$-dATP and a double stranded sequencing kit (USB).

\section{PAX2 ALLELE DELETION STUDIES}

PAX2 mutations could include a deletion of either a part or the whole PAX2 gene from one allele. Such deletions may not necessarily be detected by PCR-SSCP. To investigate whether deletions of the whole PAX2 gene had occurred, heterozygosity of the PAX2 gene was analysed in each patient by amplification of a $(\mathrm{CA})_{\mathrm{n}}$ dinucleotide repeat within intron 9 as previously described. ${ }^{30}$ Amplification of both alleles was evidence of heterozygosity.

\section{Results}

PAX2 MUTATIONAL STUDIES

The results of PCR-SSCP analysis of the PAX2 gene in 99 patients are summarised in tables 1 and 2, along with a summary of the PAX2 mutations which have previously been identified in 11 patients. One patient in this study (patient 985) was found to have an abnormal SSCP pattern in exon 2. No variations in PCR-SSCP patterns were observed in unaffected parental samples of patient 985 (fig 1A) or in 98 other subjects analysed for each exon of PAX2. To identify the PAX2 mutation in patient 985, exon 2 PCR products of patient 985 and his parents were subcloned and sequenced. Sequencing of independently derived mutant alleles from patient 985 identified a deletion of a thymidine nucleotide at position 611 in approximately $50 \%$ of clones (fig $1 \mathrm{~B}$ ). Normal alleles were found in the remainder of the clones from patient 985 . The observed nucleotide deletion causes a frameshift in exon 2 , and would be predicted to terminate the protein within exon 6 encoded sequences (fig 2 ). The predicted protein would be unable to bind DNA as it would lack much of the paired box DNA binding domain. This mutation would effectively result in haploinsufficiency of PAX2 because the predicted protein from the mutant allele would be non-functional. Fig 2 depicts the location of the mutation in the PAX2 protein and the frameshifted protein which is predicted to result from the mutation, and compares the effect of this mutation to other previously described mutations in PAX2.

CLINICAL REPORT OF PATIENT 985

Patient 985 is the second child born to non-consanguinous Dutch parents. $\mathrm{He}$ was 
Table 1 Comparison of phenotypes in 39 subjects examined for PAX2 mutations

\begin{tabular}{|c|c|c|c|c|c|}
\hline Patient & Age & Sex & Sporadic/germline & Category (ref) & Phenotype \\
\hline 1 & 14 & $M$ & Germline Family 1 & $A^{\star}(17)$ & $\begin{array}{l}\text { Bilateral optic nerve colobomas, bilateral renal hypoplasia, non-functional right } \\
\text { kidney, renal failure, grade IV vesicoureteric reflux, joint laxity }\end{array}$ \\
\hline 2 & 10 & $\mathbf{M}$ & Germline Family 1 & A (17) & $\begin{array}{l}\text { Bilateral optic nerve colobomas, bilateral renal hypoplasia, grade II vesicoureteric } \\
\text { reflux, trace proteinuria, high frequency hearing loss, joint laxity }\end{array}$ \\
\hline 3 & 6 & $\mathbf{M}$ & Germline Family 1 & A (17) & $\begin{array}{l}\text { Bilateral optic nerve colobomas, bilateral renal hypoplasia, end stage renal failure, } \\
\text { renal transplant, grade I vesicoureteric reflux, joint laxity, seizures }\end{array}$ \\
\hline 4 & 35 & $M$ & Germline Family 1 & A (17) & $\begin{array}{l}\text { Bilateral optic nerve colobomas, hypertension, mild proteinuria, high frequency } \\
\text { hearing loss }\end{array}$ \\
\hline 430 & 5 & $\mathbf{M}$ & Germline Family 2 & $A(16)$ & $\begin{array}{l}\text { Bilateral optic nerve colobomas, renal tubular atrophy, interstitial fibrosis, } \\
\text { proteinuria, absent right kidney, end stage renal failure, renal transplant }\end{array}$ \\
\hline 431 & 12.5 & $\mathbf{M}$ & Germline Family 2 & $A(16)$ & $\begin{array}{l}\text { Bilateral optic nerve colobomas, proteinuria, renal tubular atrophy, interstitial } \\
\text { fibrosis, glomerulosclerosis }\end{array}$ \\
\hline TRN & 5 & $\mathbf{M}$ & Sporadic & $A(18)$ & $\begin{array}{l}\text { Bilateral optic nerve colobomas, cataracts, renal tubular atrophy, glomerulosclerosi } \\
\text { mild renal dysfunction with proteinuria }\end{array}$ \\
\hline 579 & 11 & $\mathbf{M}$ & Sporadic & A (19) & $\begin{array}{l}\text { Bilateral optic nerve colobomas, bilateral renal hypoplasia, proteinuria, progressive } \\
\text { end stage renal failure, seizures }\end{array}$ \\
\hline 656 & 48 & $\mathbf{F}$ & Germline Family 3 & A (19) & $\begin{array}{l}\text { Bilateral optic disc hypoplasia, cataracts, hypertension, proteinuria, bilateral renal } \\
\text { hypoplasia, end stage renal failure, renal transplant }\end{array}$ \\
\hline 657 & 25 & $\mathbf{M}$ & Germline Family 3 & A (19) & $\begin{array}{l}\text { Left optic nerve aplasia, right optic disc hypoplasia with optic pit, chorioretinal } \\
\text { coloboma, capsular opacities, microphthalmos, retrobulbar cyst, retardation, } \\
\text { microcephaly, bilateral renal hypoplasia, end stage renal failure, renal transplant }\end{array}$ \\
\hline 2646 & 20 & $\mathbf{F}$ & Sporadic & A (19) & $\begin{array}{l}\text { Bilateral optic nerve colobomas, bilateral renal hypoplasia, end stage renal failure, } \\
\text { focal segmental glomerulosclerosis, right high frequency hearing loss, joint laxity }\end{array}$ \\
\hline 651 & 4 & $\mathrm{~F}$ & Sporadic & A (this study) & $\begin{array}{l}\text { Bilateral optic nerve colobomas, bilateral renal hypoplasia, impaired renal function, } \\
\text { right persistent hyoidal artery }\end{array}$ \\
\hline 813 & 12 & $\mathbf{M}$ & Germline Family 4 & A (this study) & $\begin{array}{l}\text { Bilateral optic nerve colobomas, acute renal failure at birth, one functional kidney, } \\
\text { growth retardation }\end{array}$ \\
\hline 814 & 33 & $\mathbf{F}$ & Germline Family 4 & A (this study) & $\begin{array}{l}\text { Bilateral optic nerve colobomas, bilateral renal hypoplasia, renal insufficiency from } \\
\text { age } 18 \text {, proteinuria, focal segmental hyalinosis and interstitial sclerosis }\end{array}$ \\
\hline 816 & 54 & $\mathbf{F}$ & Germline Family 4 & A (this study) & $\begin{array}{l}\text { Bilateral optic nerve colobomas, bilateral renal hypoplasia, renal insufficiency from } \\
\text { age } 33 \text {, proteinuria, end stage renal disease, renal transplant at age } 44\end{array}$ \\
\hline 906 & 28 & $\mathbf{F}$ & Germline Family 5 & A (this study) & Bilateral optic nerve colobomas, renal anomalies, renal failure \\
\hline 907 & 4 & M & Germline Family 5 & A (this study) & Bilateral optic nerve colobomas, renal anomalies \\
\hline 985 & 6 & $\mathbf{M}$ & Sporadic & A (this study) & $\begin{array}{l}\text { Bilateral optic nerve colobomas, cystic structure with no functional renal tissue on } \\
\text { right side, functional left kidney, no proteinuria, grade II-III reflux in left kidney }\end{array}$ \\
\hline 518 & 2.5 & M & Sporadic & $\mathrm{B}+$ (this study) & $\begin{array}{l}\text { Unilateral optic nerve coloboma, renal anomaly, heart defect (double outlet right } \\
\text { ventricle) }\end{array}$ \\
\hline 626 & $?$ & $?$ & Sporadic & B (this study) & $\begin{array}{l}\text { Joubert related (Dekaban) syndrome, including chorioretinal coloboma and } \\
\text { polycystic kidneys }\end{array}$ \\
\hline 627 & $?$ & $?$ & Sporadic & B (this study) & $\begin{array}{l}\text { Joubert related (Dekaban) syndrome, including chorioretinal coloboma and } \\
\text { polycystic kidneys }\end{array}$ \\
\hline 630 & $?$ & $?$ & Sporadic & B (this study) & Arima syndrome, including coloboma and polycystic kidneys \\
\hline 633 & $?$ & $?$ & Sporadic & B (this study) & Arima syndrome, including coloboma and polycystic kidneys \\
\hline 615 & $?$ & $\mathbf{F}$ & Sporadic & B (this study) & $\begin{array}{l}\text { CHARGE association (atypical), including bilateral colobomas of iris and optic } \\
\text { nerve }\end{array}$ \\
\hline 783 & 2.5 & $\mathbf{M}$ & Sporadic & B (this study) & $\begin{array}{l}\text { Bilateral optic nerve coloboma, macrocystic dysplastic kidneys, severe } \\
\text { neurodevelopmental delay, abdominal wall hypoplasia, undescended testes with } \\
\text { bilateral hernias, hepatic fibrosis with cholestasis and portal hypertension }\end{array}$ \\
\hline 806 & $?$ & $?$ & Sporadic & B (this study) & $\begin{array}{l}\text { Chorioretinal coloboma, renal anomalies, DiGeorge syndrome (thymus aplasia, } \\
\text { parathyroid aplasia, aortic arch anomalies) }\end{array}$ \\
\hline 711 & 14 & M & Germline Family 6 & C $\ddagger$ (this study) & $\begin{array}{l}\text { Bilateral iris and retinal colobomas, multicystic non-functional left kidney, left } \\
\text { hydronephrosis, normal renal function, intrauterine growth retardation, short statur } \\
\text { dysmorphic features }\end{array}$ \\
\hline 712 & 39 & $\mathbf{M}$ & Germline Family 6 & C (this study) & No ocular anomalies, recurrent urinary tract infections, possible vesicoureteric reflu \\
\hline 713 & 9 & $\mathbf{F}$ & Germline Family 6 & C (this study) & No ocular anomalies, multicystic kidney (resolved by 6 months) \\
\hline 652 & $?$ & $\mathbf{M}$ & Sporadic & C (this study) & $\begin{array}{l}\text { Left iris coloboma, bilateral vesicoureteric reflux, renal failure, bilateral cleft lip and } \\
\text { palate }\end{array}$ \\
\hline 659 & 37 & M & Germline Family 7 & C (this study) & $\begin{array}{l}\text { Retinitis pigmentosa, lens subluxation, lens coloboma, cataract, right renal agenesis } \\
\text { left chronic interstitial nephritis, left renal hypoplasia, renal transplant age } 27\end{array}$ \\
\hline 660 & 39 & $\mathbf{M}$ & Germline Family 7 & C (this study) & $\begin{array}{l}\text { Retinitis pigmentosa?, insulin dependent diabetes, globular aspect of lower pole of } \\
\text { left kidney }\end{array}$ \\
\hline 695 & 14 & F & Sporadic & C (this study) & $\begin{array}{l}\text { Bilateral iris and retinal coloboma, vesicoureteric reflux, renal failure, renal } \\
\text { transplant age } 11\end{array}$ \\
\hline 697 & $?$ & $\mathbf{M}$ & Sporadic & C (this study) & $\begin{array}{l}\text { Retinopathy, preauricular ear tags, vesicoureteric reflux, cystic kidneys, renal failure } \\
\text { renal transplant }\end{array}$ \\
\hline 698 & $?$ & $\mathbf{F}$ & Germline Family 8 & C (this study) & Vesicoureteric reflux \\
\hline 699 & ? & $\mathbf{F}$ & Germline Family 8 & C (this study) & $\begin{array}{l}\text { Cataracts, ataxia, peripheral neuropathy, renal agenesis, vesicoureteric reflux, renal } \\
\text { failure, renal transplant }\end{array}$ \\
\hline 792 & 2 & $\mathbf{F}$ & Sporadic & C (this study) & $\begin{array}{l}\text { Bilateral microphthalmos, cystic right kidney, left hydronephrosis, right UPJ } \\
\text { obstruction, seizures, abnormal EEG }\end{array}$ \\
\hline 847 & 8 & $\mathbf{M}$ & Sporadic & C (this study) & $\begin{array}{l}\text { Bilateral microphthalmos, microcorneas, persistent primary hyperplastic vitreous } \\
\text { body, orbital cysts, mentally retarded, nephronophthisis, atrophic renal tubules, } \\
\text { glomerulosclerosis, hyaline deposits, microtestis, large malformed ears }\end{array}$ \\
\hline 544 & 9 & $\mathbf{M}$ & Germline Family 9 & DS (this study) & Unilateral renal agenesis \\
\hline
\end{tabular}

^Renal-coloboma syndrome.

†Optic nerve coloboma associated or chorioretinal coloboma associated syndrome.

$\ddagger$ Syndrome with renal and eye abnormalities atypical of renal-coloboma syndrome.

§Kidney abnormalities only. $\mathrm{ND}=$ not done. $\mathrm{UN}=$ uninformative. $\mathrm{P}=$ promoter.

born after 36 weeks' gestation, with a birth weight of $2430 \mathrm{~g}$. At 6 weeks he was evaluated for failure to thrive, at which time a raised creatinine level $(121 \mu \mathrm{mol} / \mathrm{l})$ was found. Renal ultrasound showed a cystic structure on the right side, with no recognisable kidney. The left kidney appeared normal, except for dilatation of the upper pole. A renal scan showed a functional left kidney with delay in excretion, but no functional renal tissue was present on the right side. A voiding cystogram showed a normal bladder with grade II-III reflux on the left side. 
Table 1 Continued

\begin{tabular}{|c|c|c|c|}
\hline Karyotype & Heterozygosity of $P A X 2$ & Exons analysed & Mutation \\
\hline Normal & Heterozygous & $1-5$ & Exon 5 \\
\hline ND & Heterozygous & $1-5$ & Exon 5 \\
\hline ND & Heterozygous & $1-5$ & Exon 5 \\
\hline ND & Heterozygous & $1-5$ & Exon 5 \\
\hline Normal & Heterozygous & $1-5$ & Exon 2 \\
\hline Normal & Heterozygous & $1-5$ & Exon 2 \\
\hline $\mathrm{t}(10 ; 13)(\mathrm{q} 24 ; \mathrm{q} 12.3)$ & Heterozygous & ND & Translocation \\
\hline Normal & Heterozygous & $1-12$ & Exon 2 \\
\hline ND & Heterozygous & $1-4,7-12$ & Exon 2 \\
\hline Normal & Heterozygous & $1-10,12$ & Exon 2 \\
\hline Normal & Heterozygous & $1-10$ & Exon 2 \\
\hline ND & Heterozygous & $1-12, P$ & No \\
\hline Normal & Heterozygous & $1-12, P$ & No \\
\hline Normal & Heterozygous & $1-10,12$ & No \\
\hline Normal & Heterozygous & $1-10,12$ & No \\
\hline $\begin{array}{l}\text { ND } \\
\text { ND } \\
\text { ND }\end{array}$ & $\begin{array}{l}\text { ND } \\
\text { ND } \\
\text { Heterozygous }\end{array}$ & $\begin{array}{l}1-12, \mathrm{P} \\
1-12, \mathrm{P} \\
2,5,8,10-12, \mathrm{P}\end{array}$ & $\begin{array}{l}\text { No } \\
\text { No } \\
\text { Exon } 2\end{array}$ \\
\hline ND & Heterozygous & $1-10$ & No \\
\hline ND & ND & $1-4,6-12, \mathrm{P}$ & No \\
\hline ND & ND & $1-12, P$ & No \\
\hline $\begin{array}{l}\text { ND } \\
\text { ND } \\
\text { ND }\end{array}$ & $\begin{array}{l}\text { ND } \\
\text { ND } \\
\text { ND }\end{array}$ & $\begin{array}{l}1-11 \\
1-12, P \\
1-10\end{array}$ & $\begin{array}{l}\text { No } \\
\text { No } \\
\text { No }\end{array}$ \\
\hline ND & UN & $1-10$ & No \\
\hline ND & Heterozygous & $1-12, P$ & No \\
\hline ND & $\mathrm{UN}$ & $1-10$ & No \\
\hline $\begin{array}{l}\text { ND } \\
\text { ND } \\
\text { Normal }\end{array}$ & $\begin{array}{l}\text { UN } \\
\text { UN } \\
\text { UN }\end{array}$ & $\begin{array}{l}1-9 \\
1-9,12, \mathrm{P} \\
1-10,12, \mathrm{P}\end{array}$ & $\begin{array}{l}\text { No } \\
\text { No } \\
\text { No }\end{array}$ \\
\hline ND & ND & $1-8,10,12$ & No \\
\hline ND & ND & $1-12, P$ & No \\
\hline ND & ND & $1-12, P$ & No \\
\hline ND & ND & $1-10$ & No \\
\hline $\begin{array}{l}\text { ND } \\
\text { ND }\end{array}$ & $\begin{array}{l}\text { ND } \\
\text { ND }\end{array}$ & $\begin{array}{l}1-10 \\
1-9\end{array}$ & $\begin{array}{l}\text { No } \\
\text { No }\end{array}$ \\
\hline ND & ND & $1-12, P$ & No \\
\hline Normal & ND & $1-12, P$ & No \\
\hline ND & Heterozygous & $1-11$ & No \\
\hline
\end{tabular}

An intravenous pyelogram showed a visible left kidney without obvious abnormalities. Urine analysis was normal with no proteinuria and occasional leucocytes. At 3 months of age the patient had a right nephrectomy and vesicostomy. Ocular abnormalities included a convergent squint and abnormal optic nerves with colobomas. The fundi were otherwise normal, as were lens tissues and anterior chambers.
Additional investigations, including MRI scan, $x$ ray of the spine, ECG, BAEP, and VEP all yielded normal results. His general health and development have been normal, except for recurrent parotitis, which was attibuted to Furadantin maintenance therapy.

\section{Discussion}

Five different PAX2 mutations have been identified in 12 subjects with renal-coloboma syndrome $^{16-19}$ (this study). Most people with PAX2 mutations have been found to suffer from optic nerve colobomas, renal hypogenesis, and renal dysfunction, although some patients had one or more additional anomalies, including vesicoureteric reflux (VUR), high frequency hearing loss, CNS anomalies, other eye abnormalities, and joint laxity (table 1). The patient with a PAX2 mutation in this study (patient 985) presented with bilateral optic nerve colobomas, a functional left kidney, a cystic structure in place of the right kidney, and vesicoureteric reflux, and was found to have a deletion of a thymidine nucleotide at position 611 in exon 2 of PAX2 ${ }^{17}$ The identification of a PAX2 mutation in this patient concurs with the identification of a PAX2 mutation in a family with optic nerve colobomas, vesicoureteric reflux, and renal anomalies in a previous study, ${ }^{17}$ and suggests that in a small sample of patients the position of the mutation within the PAX2 gene does not necessarily correlate with the occurrence of vesicoureteric reflux; patient 985 in this study had a mutation in exon 2, while the three sibs in the previous study, who had vesicoureteric reflux ${ }^{17}$ were found to have a mutation in exon 5 of PAX2.

The phenotype associated with PAX2 mutations is highly variable, even in patients with identical mutations. ${ }^{19}$ In view of the marked variability in expression of the PAX2 mutant phenotype, we have hypothesised that PAX2 mutations may cause eye, renal, or ureteric abnormalities in isolation. In this study we examined 99 patients with either colobomas or microphthalmia in isolation or colobomas and associated anomalies for PAX2 mutations by PCR-SSCP analysis. We found that novel PAX2 sequence variants were uniformly absent in these patients, except for the identification of a PAX2 mutation in patient 985. A full list of the phenotypic abnormalities found in 110 patients analysed for PAX2 mutations, including previously reported patients, is given in tables 1 and 2. The identification of the spectrum of phenotypic features in patients who have renal-coloboma syndrome will be clinically useful as a basis for assessing which patients to screen for PAX2 mutations.

We have previously reported that PAX2 mutations are absent in patients with isolated familial vesicoureteric reflux. ${ }^{33}{ }^{34}$ These findings suggest that PAX2 mutations may not be found in patients with ureteric anomalies in isolation. However, it remains to be determined whether PAX2 mutations will be associated with isolated renal abnormalities. PAX2 mutations are associated with renal hypoplasia, tubular atrophy, glomerulosclerosis, progressive renal failure, and vesicoureteric reflux 
Table 2 Absence of PAX2 mutations in 71 subjects or families with predominantly eye abnormalities

\begin{tabular}{|c|c|c|}
\hline Phenotype & Inheritance & $\begin{array}{l}\text { No of patients } \\
\text { analysed (no of } \\
\text { families) }\end{array}$ \\
\hline Optic nerve hypoplasia (or coloboma) & Sporadic & 5 \\
\hline Optic nerve hypoplasia (or coloboma) & Inherited & $2(1)$ \\
\hline Optic nerve coloboma with cataract & Sporadic & 1 \\
\hline \multicolumn{3}{|l|}{ Bilateral optic nerve colobomas with } \\
\hline & Sporadic & 1 \\
\hline \multicolumn{3}{|l|}{ Bilateral optic nerve colobomas (with } \\
\hline hypospadias in 2 subjects) & Inherited & $4(1)$ \\
\hline Microphthalmos & Sporadic & 26 \\
\hline Microphthalmos & Inherited & $13(6)$ \\
\hline Microphthalmos with optic nerve hypoplasia & Sporadic & 1 \\
\hline $\begin{array}{l}\text { Microphthalmos with iris, choroid, retina and } \\
\text { optic disc coloboma (and VUR in } 1 \\
\text { subject) }\end{array}$ & $\begin{array}{l}\text { Inherited }{ }^{\star} \text { (mother had only } \\
\text { pigment deposition on retina } \\
\text { in line with colobomatous } \\
\text { cleft) }\end{array}$ & $3(1)$ \\
\hline $\begin{array}{l}\text { Microphthalmos with bilateral iris and retinal } \\
\text { colobomas and pupil coloboma }\end{array}$ & Sporadic ${ }^{\star}$ & 1 \\
\hline \multicolumn{3}{|l|}{$\begin{array}{l}\text { Unilateral chorioretinal coloboma (plus } \\
\text { extreme contralateral microphthalmos and }\end{array}$} \\
\hline PUJ obstruction in daughter) & Inherited ${ }^{\star}$ & $2(1)$ \\
\hline Aniridia & Sporadic & 6 \\
\hline Aniridia & Inherited & 0 \\
\hline \multicolumn{3}{|l|}{ Bilateral aniridia with optic nerve and foveal } \\
\hline Iris coloboma with bilateral retinal hypoplasia & Inherited ${ }^{\star}$ & $1(1)$ \\
\hline Bilateral pupil coloboma & Sporadic & 1 \\
\hline Optic neuropathy & Sporadic & 2 \\
\hline $\begin{array}{l}\text { Hypoplasia of ciliary body, choroid, retina, } \\
\text { and optic nerve }\end{array}$ & Sporadic* & 1 \\
\hline
\end{tabular}

^Subjects analysed who were heterozygous for a polymorphism in PAX2.

Table 3 PAX2 PCR-SSCP primer sequences and reaction conditions

\begin{tabular}{|c|c|c|c|c|}
\hline Exon & Sequence & $\begin{array}{l}\text { Mg } \\
\text { conc }\end{array}$ & $T m$ & $\begin{array}{l}\text { Product } \\
\text { size (bp) }\end{array}$ \\
\hline$P^{\star}{ }^{\star} \dagger$ & 5' GGG CTC CAG CGC TGG CGA ATC ACA 3' & & & \\
\hline PRf & 5' CGC CGC CGT GTC GCC TCT CAA ACT 3' & 1.5 & 65 & 791 \\
\hline $1 \mathrm{~F}$ & 5' GTT CAC TCA TCC TCC CTC CCC CAC C $3^{\prime}$ & & & \\
\hline iR & $5^{\prime}$ GGA GCC GGG CGC GGG TAC TC $3^{\prime}$ & 1.5 & 60 & 179 \\
\hline $2 \mathrm{~F}$ & 5' CTG TGT GTG GGG TGT TGT GTT 3' & & & \\
\hline $2 \mathbf{R}$ & 5' AAG GCG TCT CTC CCG GGA CAG CTG C 3' & 2.0 & 58 & 246 \\
\hline $3 \mathrm{~F}$ & 5' TGA CCG GCT TTC CCG GCG CA 3' & & & \\
\hline $3 R$ & 5' GAG GAA GCT GGA GTC CAG CC 3' & 1.25 & 60 & 262 \\
\hline $4 \mathrm{~F}$ & 5' TGG AGC TGC GTT TCC TGC CTT 3' & & & \\
\hline $4 \mathrm{R}$ & 5' AAT TGG CCG GAA TAG CAG TGG 3' & 3.0 & 61 & 210 \\
\hline $4 S$ & 5' CGG AAT AGG AGT GGC ATT TGA 3' & & & \\
\hline $4 \mathrm{~A}$ & 5' CTC TAG GTG GGA TCT GGT TT 3' & 1.25 & 58 & 181 \\
\hline $5 \mathrm{~F}$ & 5' TGA TGC CAT TTC CTC CTT CC $3^{\prime}$ & & & \\
\hline $5 \mathbf{R}$ & 5' GCC ACA CCT CTT CCC TCC T 3' & 1.0 & 60 & 175 \\
\hline $6 \mathrm{~F}$ & 5' CAG TGT TTG TCT GTC TCT TAT TTG CT 3' & & & \\
\hline 6R & 5' ATG TTC CCT CTG GCC CTC A 3' & 1.0 & 52 & 121 \\
\hline $7 \mathrm{~F}$ & 5' CGC CCC GAG TGT CCA TGT GTT 3' & & & \\
\hline $7 \mathbf{R}$ & 5' TAC TTC TGC AAG CAG AAA GCT CCC T 3' & 1.5 & 58 & 234 \\
\hline $8 \mathrm{~F}$ & 5' CCT TTC TCT GTG CGT GCA TCA ATA GA 3' & & & \\
\hline $8 \mathrm{R}$ & $5^{\prime}$ GGC ACC CTC CAC TGA ACG CAG 3' & 1.5 & 58 & 227 \\
\hline 9F & 5' CCC TTC CCC TTT GTG TTT TT 3' & & & \\
\hline $9 \mathrm{R}$ & 5' AGG CAG CTG CAG CAT TGT C $3{ }^{\prime}$ & 1.5 & 60 & 151 \\
\hline $10 \mathrm{~F}$ & 5' CCC CTC CCT GCA AAC CAC 3' & & & \\
\hline $10 \mathrm{R}$ & 5' CGC TGT GAG GGC CAT GAC $3^{\prime}$ & 2.0 & 57 & 150 \\
\hline $11 \mathrm{~F}$ & 5' GCA GGC GTC ACA TCC CCA CTC $3 '$ & & & \\
\hline $11 \mathrm{R}$ & $5^{\prime}$ CCG GCC ACC AGG TGG CGT A $3^{\prime}$ & 1.5 & 52 & 148 \\
\hline $12 \mathrm{~F}$ & $5^{\prime}$ ATG TGG AGG CCG AAG CTG $3^{\prime}$ & & & \\
\hline $12 \mathrm{R}$ & $5^{\prime}$ TCT GAC CCA GCC CAT TCT TCT $3^{\prime}$ & 1.0 & 60 & 163 \\
\hline
\end{tabular}

${ }^{\star} \mathrm{P}$, promoter.

$\dagger \mathrm{F}$, forward primer.

$\ddagger R$, reverse primer.

(table 1). One of us (KD) has recently identified a PAX2 missense mutation in a large pedigree presenting with renal hypoplasia and mild ocular anomalies (submitted). All affected members had renal involvement with only mild optic disc anomalies, which in most cases did not affect visual acuity. These findings emphasise the importance of an eye examination in patients presenting with renal hypoplasia.

Mutations in PAX2 appear predominantly to cause optic nerve abnormalities and renal hypoplasia in patients with renal-coloboma syndrome. It is not known what genes might be responsible for optic nerve colobomas in isolation, or for the variable association of colobomas and urogenital anomalies in renátcoloboma-like syndromes. Several genes couk be candidates, including the $\mathrm{CHX} 10$ gene which has been found to cause microphthalmoa in mice, ${ }^{35}$ or the putative gene for cat ese syndrome on chromosome 22 , which has yet to be identified.

That we did not detect PAX2 mutations क्ष 98 patients who had either ocular abnorma ties in isolation or eye defects with associated abnormalities suggests that PAX2 mutations do not cause colobomas in isolation, microptithalmia or retinal anomalies, or syndromes gf coloboma, renal anomalies, and atypical assoé ated features. In agreement with these resultsin family with dominant inheritance of optic p\#s was analysed for PAX2 mutations by SSCP $\frac{3}{\mathrm{\omega}}$ exons 1-10 of PAX2 and was not found to hacy PAX2 sequence variants. ${ }^{36}$ Included among the patients analysed in this study were two patients with a subtype of Joubert syndrom\&, referred to as Dekaban syndrome ${ }^{9}$ (choriore nal coloboma with cerebellar vermis aplasia and polycystic kidneys) (patients 626 ard 627), and also two patients with Arinta syndrome ${ }^{37}$ (blindness, cerebellar vermis agemesis, polycystic kidneys, hepatic fibrosis) (pq tients 630 and 633). Several other syndromes, for example acro-renal-ocular syndrome branchio-oculo-facial syndrome, ${ }^{13}$ and cat eqe syndrome, ${ }^{14}$ may include optic nerve colobsmas as part of the phenotype, but we have no examined such patients for PAX2 mutations $\frac{0}{\mathrm{D}}$

In this study three families had a syndrone that was considered to be identical to ren coloboma syndrome (patients 651, 813, 814, 816,906 , and 907), yet PAX2 mutations we not identified in these patients. One explani:tion for the lack of PAX2 mutations is thit there is a low level of PCR-SSCP sensitivity our screening procedure, and that certain typês of mutations are not being detected. ${ }^{38}$ How ever, our experience with the PCR-SSG method has shown that it is sufficien sensitive (85-100\% detection rate) to identify polymorphisms in DNA sequences with a GE

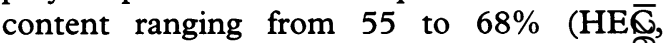
unpublished data). This level of detection of polymorphisms by PCR-SSCP is in agreemegt with observations made by others. ${ }^{39}$ Alternative explanations as to why only seven out of $\overline{0}$ families with renal-coloboma syndrome wefe identified as having PAX2 mutations are that the mutations were in sequences that we havife not yet analysed, or that there are heterogensous causes for the association of optic nerpe colobomas and renal anomalies. Mutations genes either affecting the expression of PAX? (giving rise to insufficient PAX2 expression) Or in downstream targets of the PAX2 protem might be predicted to give rise to a syndron with features closely resembling that of rensi coloboma syndrome.

We conclude from this study that PAX2 mutations are unlikely to be common in patients with ocular colobomas in isolation or in patients with ocular colobomas and associated anomalies. However, patients with typical 
A

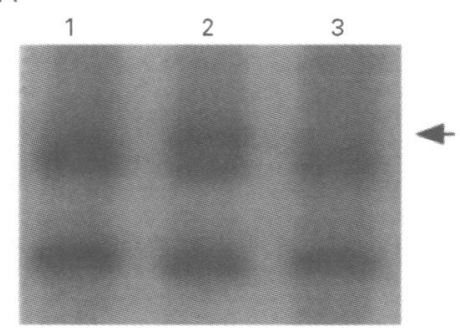

B

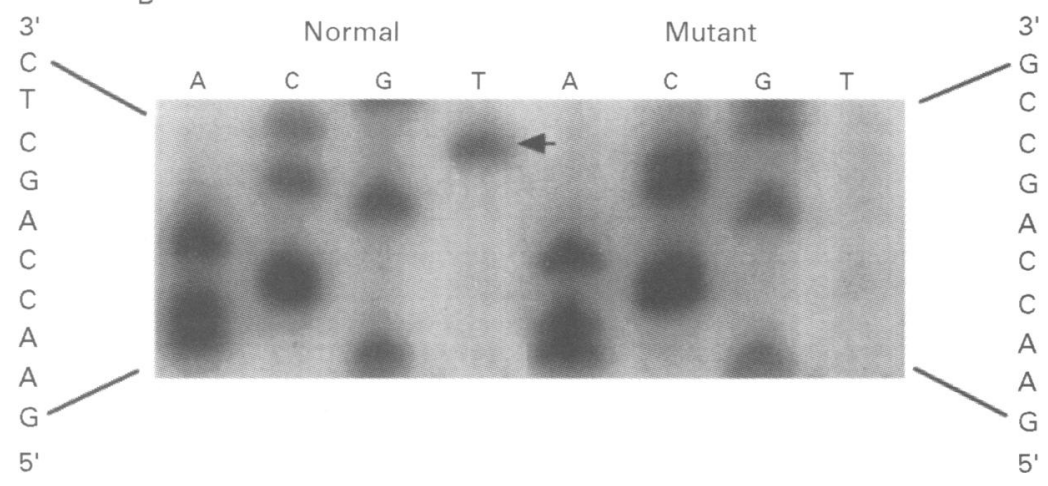

Figure 1 Identification of the mutation in exon 2 of PAX2. (A) SSCP analysis of exon 2. The primers and conditions for amplification are described in table 3. Lane 1, mother's DNA. Lane 2, affected subject's DNA (patient 985). Lane 3, father's DNA. An additional band is present in lane 2 (arrow) in DNA from the affected subject. The pattern observed in the mother's and father's DNA is normal. (B) Sequence of the normal and mutant alleles. Sequences were obtained from subcloned PCR products corresponding to normal and mutant alleles from patient 985 . Seven independent clones in pbluescript were identified that contained an identical mutation, and a further seven clones from the affected patient's $D N A$ contained normal PAX2 sequence. A single base deletion (arrow) was observed in the affected subject's DNA.
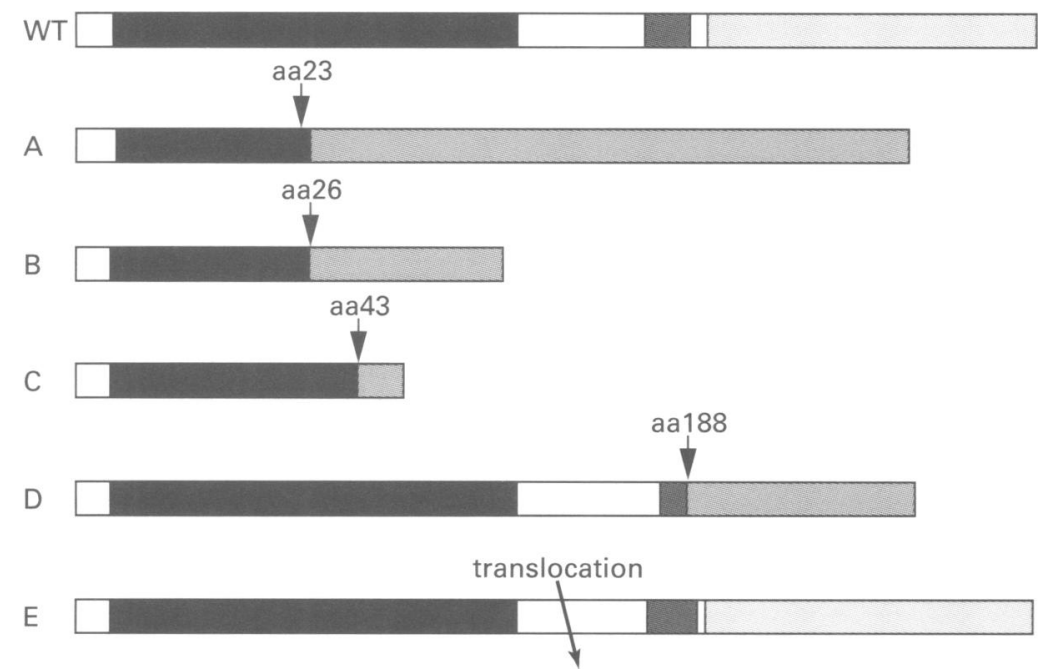

Figure 2 Depiction of the location of mutations which have been identified to date within $P A X 2$ and the effect of these mutations on the predicted PAX2 protein products. The boxed segments represent the protein domains. The conserved paired box domain (black portion), the octapeptide sequence (dark grey portion), and the transactivation domain (light grey portion) are shown. Frameshifted sequences in the mutated protein are depicted by mid grey portions. WT is the normal wild type protein. $A$ is the mutation identified in the present study, with a single base deletion at amino acid 23 which results in a frameshift and a truncated protein. $B, C, D$, and $E$ depict the predicted protein of previously identified mutations..$^{16.19}$ The mutation in $B$ is a single base insertion at amino acid position $26^{16}{ }^{19}$ The mutation in $C$ is a 22 base deletion at amino acid position $43 .{ }^{9}$ The mutation in $D$ is a single base insertion at amino acid position 188 in the octapeptide domain. "All of these point mutations are predicted to cause a frameshift and truncated protein product. Note, that the predicted proteins in $A, B$, and $C$ lack much of the paired DNA binding domain (black), as well as the octapeptide domain and COOH-terminal portions of PAX2 protein, whereas the protein in D lacks only the octapeptide and remaining COOH-terminal portions. The mutation in $E$ is a translocation $(10 ; 13)(q 24 ; q 12.3)$ with a breakpoint in the middle of the PAX 2 gene, ${ }^{1 A}$ probably within either intron 3 or 4 , which would result in an abnormal, and possibly truncated protein. renal-coloboma syndrome are very likely to have PAX2 mutations.

We gratefully acknowledge Drs $\mathrm{R}$ McInnes, A Levin, R J M Gardner, E Haan, P Bitoun, I Gupta, J P Fillastre, R Nelson, L Market, D Clark, J Pellegrino, R Koenekoop, A Schinzel, and Y Pirson for the provision of patient samples for analysis in this study. We also thank Drs. H Cruysberg, L Monnens, and R De Boer for additional clinical information on the affected patient. This research was funded by the Health Research Council of New Zealand, the Cancer Society of New Zealand, and the New Zealand Lotteries Grants.

1 Pagon RA. Ocular coloboma. Surv Ophthalmol 1981;25:223-36.

2 Reiger G. Zum krankheitsbild der Handmannschen sehnervenanomalie: windenbluten- (Morning Glory) syndrom? Klin Mbl Augenheilkd 1977;1 170:697-706.

3 Karcher H. Zum Morning Glory syndrom. Klin Mbl Augenheilkd 1979;175:835-40.

4 Weaver RG, Cashwell LF, Lorentz W, Whiteman D, Geisinger KR, Ball M. Optic nerve coloboma associated with renal disease. Am $\mathcal{f}$ Med Genet 1988;29:597-605.

5 Bron AJ, Burgess SE, Awdry PN, Oliver D, Arden G. Papillo-renal syndrome. An inherited association of optic disc dysplasia and renal disease. Report and review of the literature. Ophthal Paediatr Genet 1989;10:185-98.

6 Legros Y, Carrenard JR, Cortes FM, Sanchez JM. Sindrome de "ojo de gato" con agenesia renal derecha. Aportacion de un caso y revision de la literatura. An Esp Pediatr 1992;36: 317-19.

7 Schimmenti LA, Pierpont ME, Carpenter BLM, Kashtan CE, Johnson MR, Dobyns WB. Autosomal dominant optic nerve colobomas, vesicoureteral reflux, and renal anomalies. Am $\mathcal{F}$ Med Genet 1995;59:204-8.

8 Pagon RA, Graham JM Jr, Zonana J, Yong SL. Coloboma, congenital heart disease, and choanal atresia with multiple anomalies: CHARGE association. $\mathcal{F}$ Pediatr 1981;99:223-7.

9 Saraiva JM, Baraitser M. Joubert syndrome: a review. $A m \mathcal{F}$ Med Genet 1992;43:726-31.

10 Verloes A, Lambotte C. Further delineation of a syndrome of cerebellar vermis hypo/aplasia, oligophrenia, congenital ataxia, coloboma, and hepatic fibrosis. Am $\mathcal{F}$ Med Genet 1989;32:227-32

11 Halal F, Homsy M, Perreault G. Acro-renal-ocular syndrome: autosomal dominant thumb hypoplasia, renal ectopia, and eye defect. Am f Med Genet 1984;17:753-62.

12 Sorsby A. Congenital coloboma of the macula, together with an account of the familial occurrence of bilateral macular coloboma in association with apical dystrophy of hands and feet. Br f Ophthalmol 1935;19:65-90.

13 Richardson E, Davison C, Moore A. Colobomatous microphthalmia with midfacial clefting: part of the spectrum of pranchio-oculo-facial syndrome? Ophthal Genet 1996;17: $59-65$

14 Schinzel A, Schmid W, Fraccaro M, et al. The 'cat eye syndrome': dicentric small marker chromosome probably derived from a 22 (tetrasomy 22pter;q11) associated with a characteristic phenotype. Report of 11 patients and delineation of the clinical picture. Hum Genet 1981;57:148-58.

15 Marles SL, Chodirker BN, Greenberg CR, Chudley AE. Evidence for Ritscher-Schinzel syndrome in Canadian native Indians. Am $\mathcal{F}$ Med Genet 1995;56:343-50

16 Sanyanusin P, McNoe LA, Sullivan MJ, Weaver RG, Eccles MR. Mutation of $P A X 2$ in two siblings with renalcoloboma syndrome. Hum Mol Genet 1995;4:2183-4.

17 Sanyanusin P, Schimmenti LA, McNoe LA, et al. Mutation of the $P A X 2$ gene in a family with optic nerve colobomas, renal anomalies and vesicoureteral reflux. Nat Genet 1995; 9:358-64.

18 Narahara K, Baker E, Ito S, et al. Localisation of a $10 \mathrm{q}$ breakpoint within the $P A X 2$ gene in a patient with a de breakpoint within the $P A X 2$ gene in a patient with a de novo $t(10 ; 13)$ translocation and optic nerve
renal disease. 7 Med Genet 1997;34:213-16.

19 Schimmenti LA, Cunliffe HE, McNoe LA, et al. Further delineation of the renal-coloboma syndrome in patients with extreme variability of phenotype and identical $P A X 2$ mutations. Am $\mathcal{f}$ Hum Genet 1997;60:869-78.

20 Azuma N, Nishina S, Yanagisawa H, Okuyama T, Yamada M. PAX6 missense mutation in isolated foveal hypoplasia. Nat Genet 1996;13:141-2.

21 Martha A, Strong LC, Ferrell RE, Saunders GF. Three novel aniridia mutations in the human PAX6 gene. Hum Mutat 1995;6:44-9.

22 Strachan T, Read AP. PAX genes. Curr Opin Genet Dev 1994;4:427-38.

23 Dressler GR, Deutsch U, Chowdhury K, Nornes HO, Gruss P. Pax2, a new murine paired-box-containing gene and its expression in the developing excretory system. Development expression in the deve
$1990 ; 109: 787-95$.

24 Nornes HO, Dressler GR, Knapik EW, Deutsch U, Gruss P. Spatially and temporally restricted expression of $\operatorname{Pax} 2$ during murine neurogenesis. Development 1990;109:797-809.

25 Eccles MR, Wallis LJ, Fidler AE, Spurr NK, Goodfellow PJ, Reeve AE. Expression of the PAX2 gene in human fetal kidney and Wilms' tumor. Cell Growth Diff 1992;3:279-89.

26 Keller SA, Jones JM, Boyle A, et al. Kidney and retinal defects $(K r d)$, a transgene-induced mutation with a deletion of mouse chromosome 19 that includes the Pax2 locus. Genomics 1994;23:309-20.

27 Favor J, Sandaluche R, Neuhauser-Klaus A, et al. The mouse Pax $2^{\text {1Neu }}$ mutation is identical to a human PAX2 
mutation in a family with renal-coloboma syndrome and results in developmental defects of brain, ear, eye and kidney. Proc Natl Acad Sci USA 1996;93:13870-5.

28 Torres M, Gomez-Pardo E, Dressler GR, Gruss P. Pax2 controls multiple steps of urogenital development. Development 1995;121:4057-65.

29 Torres M, Gomez-Pardo E, Gruss P. Pax2 contributes to inner ear patterning and optic nerve trajectory. Development 1996;122:3381-91.

30 Sanyanusin P, Norrish JH, Ward TA, Nebel A, McNoe LA, Eccles MR. Genomic structure of the human $P A X 2$ gene. Genomics 1996;35:258-61.

31 Sambrook J, Fritsch EF, Maniatis T. Molecular cloning: a laboratory manual. 2nd ed. New York: Cold Spring Harbor Laboratory Press, 1989.

32 Orita M, Suzuki Y, Sekiya T, Hayashi K. Rapid and sensitive detection of point mutations and DNA polymorphisms using the polymerase chain reaction. Genomics 1989;5:8749.

33 Choi KL, McNoe LA, French MC, Guilford PJ, Eccles MR Absence of PAX2 gene mutations in patients with primary familial vesicoureteric reflux. $\mathcal{F}$ Med Genet 1998;35:338-9.
34 Eccles MR, Bailey RR, Abbott GD, Sullivan MJ. Unravelling the genetics of vesicoureteric reflux: a common familial disorder. Hum Mol Genet 1996;5:1425-9.

35 Burmeister M, Novak J, Liang MY, et al. Ocular retardation mouse caused by Chx10 homeobox null allele: impaired retinal progenitor proliferation and bipolar cell different tion. Nat Genet 1996;12:376-84.

36 Stefko ST, Campochiaro P, Wang P, Li Y, Zhu D, Trabouts E. Dominant inheritance of optic pits. Am $\mathcal{F}$ Ophthalrepl 1997;124:112-13.

37 Matsuzaka T, Sakuragawa N, Nakayama H, Sugai K, Koh $\mathbb{\Phi}_{0}$ Y, Arima M. Cerebro-oculo-hepato-renal syndrome (Afi ma's syndrome): a distinct clinicopathological entity Child Neurol 1986;1:338-46.

38 Axton R, Hanson I, Danes S, Sellar G, van Heyningen $\mathbb{Z}_{\text {, }}$ Prosser J. The incidence of PAX6 mutation in patients wi simple aniridia: an evaluation of mutation detection in $\bar{\alpha} 2$ cases. F Med Genet 1997;34:279-86.

39 Hayashi K. PCR-SSCP: a simple and sensitive method detection of mutations in the genomic DNA. PCR Metheds and Applications 1991;7:34-8. 